\title{
The effect of passive leg-raising maneuver on hemodynamic stability during anesthesia induction for adult cardiac surgery
}

This article was published in the following Dove Press journal:

Integrated Blood Pressure Control

\author{
Solmaz Fakhari \\ Eissa Bilehjani \\ Haleh Farzin \\ Hojjat Pourfathi \\ Mohsen Chalabianlou \\ Cardiovascular Research Center, \\ Faculty of Medicine, Tabriz University \\ of Medical Sciences, Tabriz, Iran
}

\begin{abstract}
Introduction: Some cardiac patients do not tolerate the intravenous fluid load commonly administered before anesthesia induction. This study investigated preinduction passive legraising maneuver (PLRM) as an alternative method to fluid loading before cardiac anesthesia. Methods and materials: During a 6-month period, 120 adult elective heart surgery patients were enrolled in this study and allocated into 2 groups: PLRM group vs control group $(n=60)$. Anesthesia was induced using midazolam, fentanyl, and cisatracurium. Initially, $250 \mathrm{~mL}$ of fluid was administrated intravenously in all of patients before anesthesia induction. Then in the PLRM group, PLRM was performed starting 2 minutes before anesthesia induction and continued for 20 minutes after tracheal intubation. In the control group, anesthesia was induced in a simple supine position. Heart rate, invasive mean arterial blood pressure (MAP), and central venous pressure (CVP) were recorded before PLRM, before anesthetic induction, before laryngoscopy, and at 5, 10, and 20 minutes after tracheal intubation. The hypotension episode rate (MAP $<70 \mathrm{mmHg}$ ) and CVP changes were compared between the 2 groups. The predictive value of the $\geq 3 \mathrm{mmHg}$ increase in CVP value in response to PLRM for hypotension prevention was defined. Results: Hypotension rates were lower in the PLRM group (63.3\% vs $81.6 \% ; P$-value 0.04$)$, and MAP was higher among PLRM patients immediately before anesthetic injection, before laryngoscopy, and 20 minutes after intubation, compared to the control group. PLRM increased CVP by $3.57 \pm 4.9 \mathrm{mmHg}$ (from $7.50 \pm 2.94$ to $11.05 \pm 3.55 \mathrm{mmHg}$ ), which required several minutes to reach peak value, returning to baseline after 15 minutes. This change did not correlate to subsequent MAP changes; an increase in the CVP value $\geq 3 \mathrm{mmHg}$ decreased the postinduction hypotension rate by $62.50 \%$.
\end{abstract}

Conclusion: Preinduction PLRM can provide a more stable hemodynamic status in adult cardiac surgery patients and decreases anesthesia-induced hypotension rates by $62.50 \%$. Rate of the changes in the CVP value caused by PLRM is not predictive of subsequent MAP changes. Keywords: passive leg-raising maneuver, cardiac surgery, anesthesia induction, hypotension

\section{Introduction}

Hypotension is a challenging complication of anesthesia induction, and severe hypotension during induction is a serious situation requiring rapid and timely diagnosis and treatment. Almost all anesthetic drugs suppress myocardium and induce vasodilatation, which can lead to hypotension. Additionally, hypotension during anesthesia induction is exacerbated by various factors, such as preoperative hypovolemia or decreased cardiac preload, decreased myocardial contractility, and cardiac afterload. ${ }^{1}$ Among these factors, the most common cause of hypotension is the decreased preload that is being
Correspondence: Eissa Bilehjani Cardiovascular Research Center, Tabriz University of Medical Sciences, Madan Heart Hospital, Daneshqhah Street 5I666I5573, Tabriz, Iran

Tel +989141160647

Fax +98 4II 3373950

Email isadavod@gmail.com
Integrated Blood Pressure Control 2018:1 I 57-63 
induced by anesthetic drugs. It has been demonstrated that hypovolemic patients (as in prolonged preoperative fasting, preoperative diuretics usage, untreated hypertension, and preoperative bleeding) are more susceptible to hypotension during induction of anesthesia. In clinical practice, induction agents are usually prescribed at slow injection rates and modified doses to prevent severe hypotension.

An intravascular fluid load $(5-7 \mathrm{~mL} / \mathrm{kg})$ is used before anesthetic delivery to decrease the severity of anesthesiainduced hypotension. ${ }^{2,3}$ However, limited data have suggested that administration of preoperative intravenous (IV) fluids prevents hypotension during induction. ${ }^{4-6}$ The efficacy of preinduction volume expansion can be assessed by defining the heart-filling pressure or preload, which refers to the stretching rate of the ventricular myocardium at the end of the diastole and end diastolic pressures (ie, the heart-filling pressure). In the right and left ventricles, this pressure is the same in healthy individuals and is measured as the central venous pressure (CVP). Normally, stroke volume and cardiac output $(\mathrm{CO})$ are dependent on the left ventricular end diastolic pressure, and any changes in this pressure can affect the hemodynamic parameters during induction of anesthesia.

Perioperative fluid administration is an important aspect of the anesthesia clinical practice; it can decrease the severity of the anesthesia-induced hypotension. In normal patients, this fluid leaves the patient's body through postoperative diuresis (induced spontaneously or by diuretic agents). In patients with limited cardiac reserves, intravascular volume expansion may expose patients to some postoperative risks such as edema. A small fraction of intravascular volume is in the arterial system, and $75 \%$ of blood is contained in the venous system; therefore, the venous system is an important intravascular reservoir.

Theoretically, raising the lower extremities affects arterial blood flow by facilitating venous blood drainage from these extremities. In a recent research, the hemodynamic response to PLRM, considered as auto-transfusion, has attracted widespread attention. ${ }^{7}$ This auto-transfusion or translocation increases cardiac preload by $10 \%-15 \%$, and also CO in the same value, through Frank-Starling mechanism. ${ }^{8-10}$ PLRM have been reported to transport $150-300 \mathrm{~mL}$ of blood from the legs to the central blood department, ${ }^{11}$ through a process that is rapid, reversible, and applicable to clinical cases that require rapid volume expansion, such as in emergency departments or intensive care units. ${ }^{12-15}$

This hypothesis has been challenged due to the transience of the effects of PLRM. Most patients who undergo cardiac surgery have limited cardiac reserves and consume drugs with target organs in the cardiovascular system. They are highly sensitive to anesthesia induction or intravascular volume expansion, both of which can expose patients to intra- and postoperative risks. PLRM may function as a buffer that temporarily and rapidly delivers adequate blood volume from the periphery of the patient's body to the central circulation system. PLRM also increase CVP, although some studies have shown that this increase is a temporary phenomenon. ${ }^{8}$ The aim of the present research was to investigate the efficacy of PLMR to prevent or modify the rate or severity of post-anesthesia-induced hypotension during adult cardiac anesthesia.

\section{Methods and materials}

The study was performed after obtaining the approval of the local institutional ethics committee (Tabriz University of Medical Sciences; registry number: 5/4/9365-92/11/15), after registering with an Iranian randomized clinical trial organization (registry number: $201410111127 \mathrm{~N} 4$, www.irct.com), and after obtaining written preoperative informed consent from all patients. This study was conducted during a 6-month period on patients who were candidates for elective cardiac surgery at the university cardiac hospital; 120 patients, between 18 and 70 years old, with the American Society of Anesthesiologists class II, enrolled in the investigation and were separated into 2 groups randomly: a PLRM group and a control group $(n=60)$. The sample size was calculated based on a possible decreasing rate of hypotension during anesthesia induction from $30 \%$ to $10 \%$ caused by PLRM $(\alpha=0.05$ with 0.80 study power). Patients were excluded if they met the following criteria: repeated or emergency surgery, American Society of Anesthesiologists class III or IV, presence of any noncardiac disease, a left ventricular ejection fraction (LVEF) $<0.40$, recent acute myocardial infarction or onset of unstable angina, a recent unstable hemodynamic history (eg, hypotension episodes or arrhythmia requiring inotropic drugs), or initial arterial systolic blood pressure $>160 \mathrm{mmHg}$.

Premedication was oral diazepam $(10 \mathrm{mg})$ and intramuscular morphine and promethazine $(0.1$ and $0.5 \mathrm{mg} / \mathrm{kg}$, respectively) 30 minutes before the patient was transferred to the operation room. In the operating room, $250 \mathrm{~mL}$ of Ringer's solution was infused through a peripheral venous catheter for 15 minutes and continued at a rate of $10 \mathrm{~mL} / \mathrm{min}$. Using local anesthesia, a 20G catheter and a 7F double lumen central venous catheter were placed in the left radial artery and right internal jugular vein, respectively. Anesthesia was induced using fentanyl (5 $\mu \mathrm{g} / \mathrm{kg})$, midazolam $(0.1 \mathrm{mg} / \mathrm{kg})$, and cisatracurium $(0.2 \mathrm{mg} / \mathrm{kg})$. In the PLRM group, PLRM 
was performed 1-2 minutes before anesthesia induction by passive leg-raising $30^{\circ}-45^{\circ}$. In the control group, PLRM were not performed. Direct arterial and CVP, heart rate, and electrocardiography were monitored using an anesthesia hemodynamic monitor (Datascope Spectrum OR; Mindray DS USA, Inc., Mahwah, NJ, USA). Hypotension (systolic blood pressure $<100 \mathrm{mmHg}$ or MAP $<70 \mathrm{mmHg}$ ) was treated using $10 \mathrm{mg}$ of IV ephedrine boluses, and hemodynamic parameters were recorded for 20 minutes after tracheal intubation. Then, a blinded coworker recorded the hemodynamic data from the anesthesia monitoring system at marked event times. These data were direct arterial blood pressure, CVP, and heart rate at 2 minutes prior to performance of PLRM to form a baseline ( 4 minutes prior to anesthetic injection in control group); immediately before anesthetic injection; immediately before laryngoscopy; and at 5, 10, and $20 \mathrm{~min}-$ utes after tracheal intubation. Mean arterial blood pressure (MAP) below $70 \mathrm{mmHg}$ was considered hypotension.

\section{Statistical analysis}

The demographic data, drug history, type of heart disease, heart rate, arterial blood pressure, and CVP measurements were analyzed using SPSS statistical software (Version 16.0; SPSS Inc., Chicago, IL, USA). Normally distributed parametric variables, such as heart rate, arterial blood pressure, CVP, LVEF, weight, and age, were presented as standard deviation (SD) \pm mean and were compared using the Student's $t$-test for independent groups. Categorical data, such as hypotension episode, ephedrine use, drug history, gender, and type of heart disease, were presented as frequencies (percentage) and analyzed using $\chi^{2}$ or Fisher's exact tests. The significance of the changes in heart rate, arterial pressure, and CVP at various times was tested using the analysis of variance for repeated measures. A Pearson correlation test was used to define the correlation between the rate of CVP change in response to PLRM and the mean arterial blood change. The predictive value of the CVP changes in response to PLRM to prevent hypotension after anesthesia induction was determined. All comparisons were 2 -sided, and $P$-values $<0.05$ were considered statistically significant.

\section{Results}

This study included 120 patients, of whom 56.7\% (68) were male and $43.3 \%$ (52) were female. Coronary artery bypass graft surgery was the most commonly performed surgery. Demographic data and drug history, LVEF, and basic hemodynamic parameters were similar for both groups (Table 1). $\beta$-Blockers were the most commonly prescribed drug class, and all previously prescribed drugs, except for aspirin, clopidogrel, and warfarin, were continued until surgery. Cardiac diseases were also similar among both groups. The total episodes of hypotension were low for the PLRM group (38 cases [63.3\%] vs 49 cases [81.6\%]; $P$-value 0.04 ); this difference was statistically significant 20 minutes after anesthesia induction ( $P$-value 0.045; Table 1).

There was no correlation between preoperatively consumed drugs and episodes of hypotension. A temporary decrease in heart rate was observed in the PLRM group as a reaction to the maneuvers (Figure 1). PLRM improved MAP, which was higher in the PLRM group. This effect was seen immediately before anesthetic injection, at pre-laryngoscopy, and 20 minutes after tracheal intubation (Figure 2). The increase in CVP caused by PLRM required several minutes to reach the peak value, with an increase of $3.57 \pm 4.9 \mathrm{mmHg}$ (from $7.50 \pm 2.94$ to $11.05 \pm 3.55 \mathrm{mmHg}$ ) recorded immediately before laryngoscopy. This was a temporary effect and neutralized completely after 15 minutes (Figure 3). A Pearson correlation analysis showed that the CVP changes in response to PLRM did not correlate to mean arterial blood changes at any time. The positive predictive value of increased CVP $\geq 3$ $\mathrm{mmHg}$ in response to PLRM, to prevent hypotension episodes (MAP below $70 \mathrm{mmHg}$ ), was $62.50 \%$. Anesthesia induction did not have any significant effect on CVP in the control group, for whom postanesthesia MAP was significantly lower than the initial value; however, the decrease in MAP among patients in the PLRM group was not significant.

\section{Discussion}

Fluid therapy is a foundational treatment for patients with hypotension commonly employed during emergencies, anesthesia induction, and operations. ${ }^{16}$ This method must be conducted with caution and with close monitoring of the patients; in some cases, this intravascular volume expansion may expose patients to additional perioperative risks. Anesthesia induction can lead to short periods of mild hypotension, most commonly among hypovolemic patients. To prevent hypotension, anesthesiologists routinely administer a bolus of fluid $(3-5 \mathrm{~mL} / \mathrm{kg})$ intravascularly before initiating anesthesia. Patients undergoing cardiac surgery have limited cardiac reserves and are sometimes volume depleted by preoperative diuretic consumption, which can cause anesthesia-induced hypotension. It is difficult to predict the hemodynamic response of a patient to fluid administration.

PLMR transports peripheral blood from the lower extremities to the central intravascular department; thus, this strategy is commonly used for patients who require rapid fluid 
Table I Demographic, preoperative variables and hypotension rates of the patients (mean \pm SD or frequency [\%])

\begin{tabular}{|c|c|c|c|c|}
\hline Characteristic & PLRM group $(\mathrm{N}=60)$ & Control group $(\mathrm{N}=60)$ & Total $(N=120)$ & $P$-value \\
\hline Male/Female & $33 / 27$ & $35 / 25$ & $68 / 52$ & \\
\hline Age (year) mean $\pm S D$ & $54.88 \pm 12.92$ & $58.48 \pm 9.37$ & $56.68 \pm 11.38$ & 0.83 \\
\hline Weight $(\mathrm{kg})$ mean $\pm \mathrm{SD}$ & $72.23 \pm 12.06$ & $72.72 \pm 12.23$ & $72.47 \pm 12.09$ & 0.82 \\
\hline Height $(\mathrm{cm})$ mean $\pm \mathrm{SD}$ & $163.82 \pm 10.67$ & $161.52 \pm 10.54$ & $162.67 \pm 10.63$ & 0.24 \\
\hline \multicolumn{5}{|l|}{ Type of heart disease } \\
\hline CAD & $50(83.3 \%)$ & 45 (75\%) & $95(79.1 \%)$ & 0.130 \\
\hline Valvular & $8(13.3 \%)$ & $9(15 \%)$ & $17(14.2 \%)$ & \\
\hline CAD/valvular & $2(3.3 \%)$ & $\mathrm{I}(\mathrm{I} .7 \%)$ & $3(2.5 \%)$ & \\
\hline Others & I (I.7\%) & $4(6.7 \%)$ & $5(4.2 \%)$ & \\
\hline LVEF mean \pm SD & $49.55 \pm 6.57$ & $49.83 \pm 5.82$ & $49.69 \pm 6.18$ & 0.80 \\
\hline Heart rate mean \pm SD & $74.75 \pm 14.98$ & $79.07 \pm 16.86$ & $76.91 \pm 16.03$ & 0.141 \\
\hline Mean arterial blood pressure mean \pm SD & $87.18 \pm 12.94$ & $90.39 \pm 10.83$ & $88.79 \pm 11.99$ & 0.145 \\
\hline Central venous pressure mean $\pm S D$ & $7.50 \pm 2.94$ & $8.52 \pm 3.28$ & $8.01 \pm 3.14$ & 0.077 \\
\hline \multicolumn{5}{|l|}{ Drugs history } \\
\hline$\beta$-blockers & $36(60 \%)$ & $4 \mathrm{I}(68.3 \%)$ & 77 (64.2\%) & 0.447 \\
\hline ACEls & $14(23.3 \%)$ & $21(35 \%)$ & $35(29.3 \%)$ & 0.228 \\
\hline ARBs & $7(11.7 \%)$ & $13(21.7 \%)$ & $20(16.7 \%)$ & 0.220 \\
\hline Statins & $27(45 \%)$ & $34(56.7 \%)$ & $61(50.8 \%)$ & 0.273 \\
\hline Ca-blockers & $7(11.7 \%)$ & $12(20 \%)$ & $19(15.8 \%)$ & 0.317 \\
\hline \multicolumn{5}{|l|}{ Hypotension episode ${ }^{a}$} \\
\hline Pre-maneuver & $3(5 \%)$ & $2(3.3 \%)$ & $5(4.2 \%)$ & 1.0 \\
\hline Before anesthetic injection & $0(0 \%)$ & $2(3.3 \%)$ & $2(1.7 \%)$ & 0.495 \\
\hline Pre-laryngoscopy & $16(26.7 \%)$ & $25(41.7 \%)$ & $4 \mathrm{I}(34.2 \%)$ & 0.124 \\
\hline 5 minutes after intubation & $26(43.3 \%)$ & $33(55 \%)$ & $59(49.2 \%)$ & 0.273 \\
\hline 10 minutes after intubation & $28(46.7 \%)$ & $32(53.3 \%)$ & $60(50 \%)$ & 0.584 \\
\hline 20 minutes after intubation & $24(40 \%)$ & $36(60 \%)$ & $60(50 \%)$ & $0.045^{\mathrm{b}}$ \\
\hline Total hypotension episode & $38(63.3 \%)$ & 49 (8I.6\%) & $87(72.5 \%)$ & $0.040^{\mathrm{b}}$ \\
\hline
\end{tabular}

Notes: a Mean arterial blood pressure below $70 \mathrm{mmHg}$. bSignificant difference between the two groups $(P$-value $<0.05)$.

Abbreviations: ARBs, angiotensin receptor blockers; ACEls, angiotensin-converting enzyme inhibitors; CAD, coronary artery disease; PLRM, passive leg-raising maneuver.

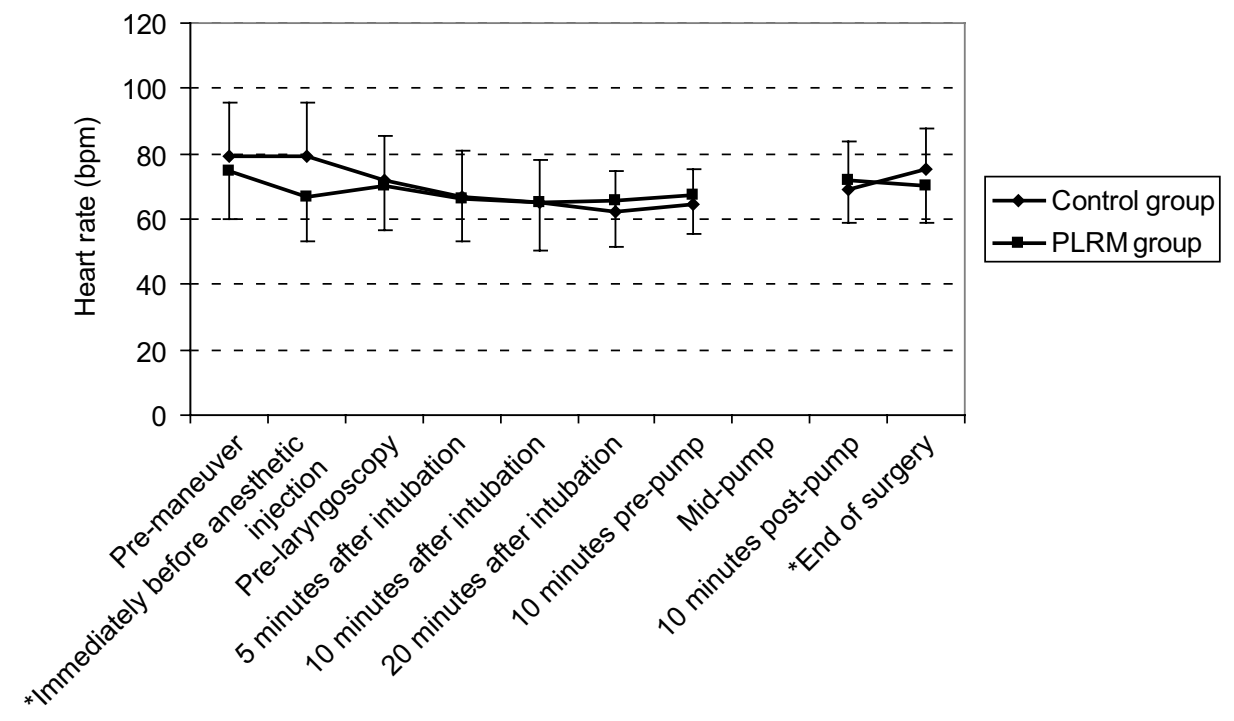

Figure I The heart rate changes in the PLRM and control groups.

Note: *Significant difference between the 2 groups $(P$-value $<0.05)$.

Abbreviation: PLRM, passive leg-raising maneuver. 


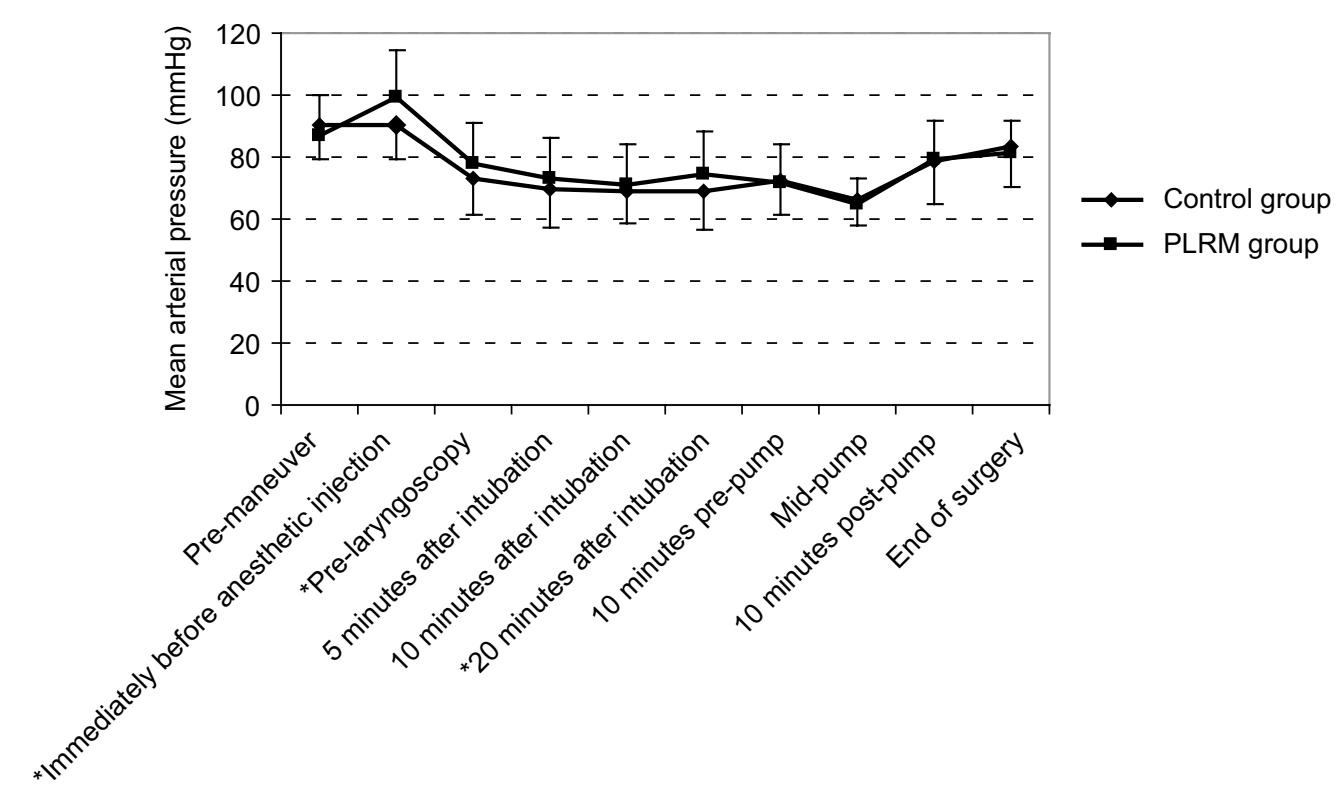

Figure 2 MAP changes in the PLRM and control groups.

Note: *Significant difference between the 2 groups $(P$-value $<0.05)$.

Abbreviation: MAP, mean arterial blood pressure; PLRM, passive leg-raising maneuver.

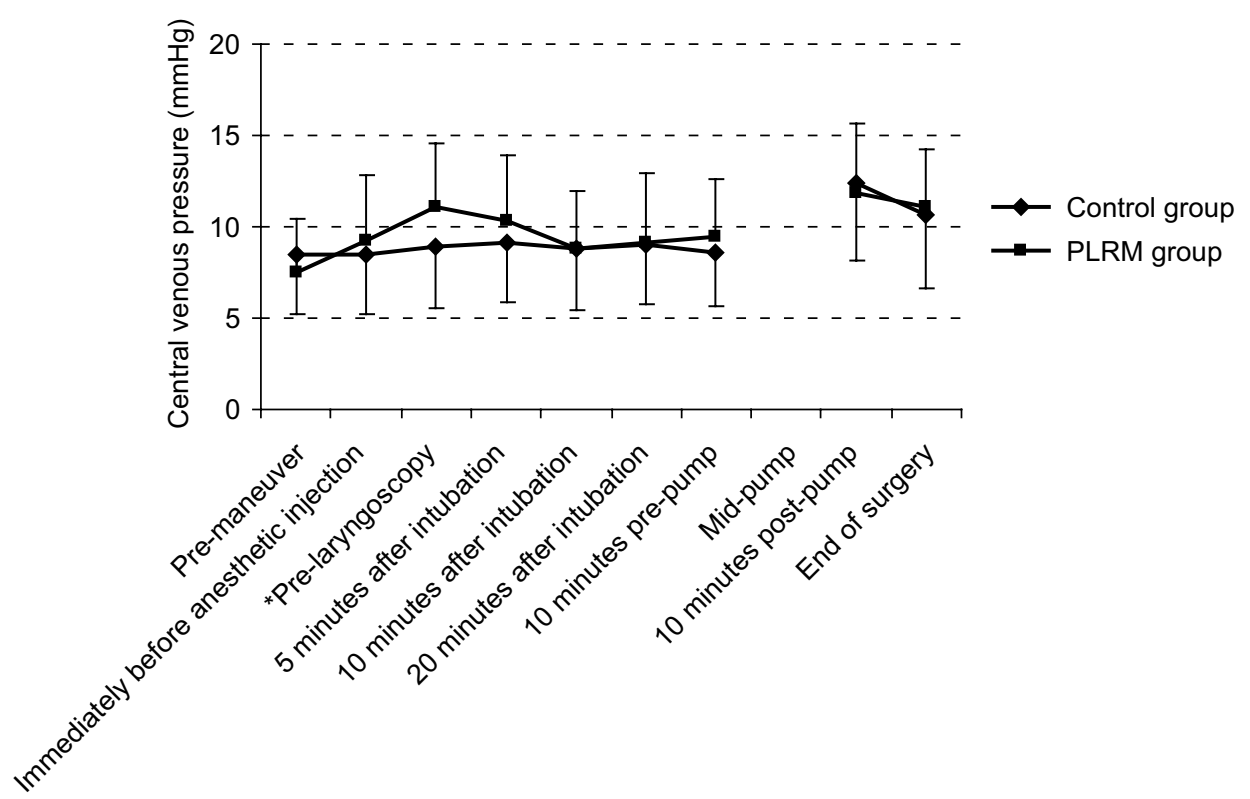

Figure 3 CVP changes in the PLRM and control groups.

Note: *Significant difference between the 2 groups $(P$-value $<0.05)$.

Abbreviations: CVP, cardiovascular pressure; PLRM, passive leg-raising maneuver.

resuscitation. Duus et $\mathrm{al}^{17}$ measured $\mathrm{CO}$ and reported that PLRM are a more valuable tool than fluid bolus for predicting patients' responsiveness to fluid. Bentzer et al ${ }^{18}$ reviewed 50 studies that investigated the diagnostic accuracy of predictive tests for fluid responsiveness among hemodynamically compromised and unstable patients, concluding that an objective increase in $\mathrm{CO}$ or related parameters after PLRM may be the most useful test for predicting patients' fluid responsiveness. Cherpanath et $\mathrm{l}^{19}$ found the same results in a meta-analysis.

In the present study, the efficacy of PLRM for producing stable hemodynamic conditions during anesthesia induction in cardiac surgery was evaluated. The lower extremities of patients were raised prior to anesthetic induction to effectively reduce hypotension rates. The effect of such maneuvers 
on CVP required several minutes to reach peak value and lasted for 15 minutes. This was in accordance with Gaffney's findings that after 7 minutes, the effect of PLRM on stroke volume and $\mathrm{CO}$ dissipated among healthy volunteers. ${ }^{8}$ Here, the peak increase in CVP was more than $4 \mathrm{mmHg}$, which agreed with Lakhal et al's ${ }^{20}$ findings that CVP changes after PLRM were at least $\geq 2 \mathrm{mmHg}$, which is required to affect patients' hemodynamic parameters.

PLRM are regularly prescribed to treat hypotension or to predict patient responses to volume expansion, although few studies have used PLRM for prophylactic purposes. ${ }^{12}$ Administration of PLRM to prevent anesthesia-induced hypotension differs from volume responsibility assessment in that all aspects of PLRM (eg, increased preload, afterload, and $\mathrm{CO}$ ) contribute to such prevention. The current study proved that PLRM help maintain hemodynamic stability, similar to the findings of $\mathrm{Yu}$ et al, ${ }^{9}$ who evaluated critically ill patients without significant cardiac disease requiring sedation with dexmedetomidine and found that PLRM reduce sedation-induced hypotension. Here, PLRM reduced heart rates for short periods, similar to Monnet et $\mathrm{al}^{12}$ and Kyriakides et al, ${ }^{21}$ who reported increased $\mathrm{CO}$ and arterial blood pressure in response to PLRM as positive responses to fluid therapy. Measuring CO using echocardiography is an operator-dependent skill that may cause study bias; therefore, the current study did not measure CO.

The head-down tilt or Trendelenburg position uses a similar mechanism to transport blood from the lower extremities to the upper body and has been employed successfully to treat or prevent hypotension during the application of some anesthetic techniques. ${ }^{22,23}$ Frost studied this position and concluded that it can help identify patients who need IV fluid after surgery; ${ }^{24}$ however, Reuter et $\mathrm{al}^{25}$ reported that this position does not improve cardiac performance. Thus, despite such controversies regarding the potential of PLRM for predicting patient volume status, the present study proved such maneuvers to be useful for reducing the prevalence or severity of hypotension during anesthesia induction.

\section{Conclusion}

Anesthesia-induced hypotension is a common complication among patients who undergo cardiac surgery. This study concluded that PLRM is a simple, rapid, safe, and effective method for preventing anesthesia-induced hypotension and helps induce anesthesia in a more stable hemodynamic state during cardiac operations.

\section{Acknowledgment}

The study was financially supported by grants from the Cardiovascular Research Center of Tabriz University of Medical Sciences.

\section{Disclosure}

The authors report no conflicts of interest in this work.

\section{References}

1. Reich DL, Hossain S, Krol M, et al. Predictors of hypotension after induction of general anesthesia. Anesth Analg. 2005;101(3):622-628.

2. Yeager MP, Spence BC. Perioperative fluid management: current consensus and controversies. Semin Dial. 2006;19(6):472-479.

3. Walsh SR, Cook EJ, Bentley R, et al. Perioperative fluid management: prospective audit. Int J ClinPract. 2008;62(3):492-497.

4. Turner RJ, Gatt SP, Kam PC, Ramzan I, Daley M. Administration of a crystalloid fluid preload does not prevent the decrease in arterial blood pressure after induction of anaesthesia with propofol and fentanyl. $\mathrm{Br}$ J Anaesth. 1998;80(6):737-741.

5. Al-Ghamdi A. Hydroxyethyl starch $6 \%$ preload does not prevent hypotension following induction with propofol and fentanyl. Middle East $J$ Anaesthesiol. 2004;17(5):959-968.

6. Corcoran T, Rhodes JE, Clarke S, Myles PS, Ho KM. Perioperative fluid management strategies in major surgery: a stratified meta-analysis. Anesth Analg. 2012;114(3):640-651.

7. Monnet X, Teboul JL. Passive leg raising: five rules, not a drop of fluid! Crit Care. 2015;19:18.

8. Gaffney FA, Bastian BC, Thal ER, Atkins JM, Blomqvist CG. Passive leg raising does not produce a significant or sustained autotransfusion effect. J Trauma. 1982;22(3):190-193.

9. Yu T, Pan C, Guo FM, Yang Y, Qiu HB. Changes in arterial blood pressure induced by passive leg raising predict hypotension during the induction of sedation in critically ill patients without severe cardiac dysfunction. Chin Med J (Engl). 2013;126(13):2445-2450.

10. Maizel J, Airapetian N, Lorne E, Tribouilloy C, Massy Z, Slama M. Diagnosis of central hypovolemia by using passive leg raising. Intensive Care Med. 2007;33(7):1133-1138.

11. Jabot J, Teboul JL, Richard C, Monnet X. Passive leg raising for predicting fluid responsiveness: importance of the postural change. Intensive Care Med. 2009;35(1):85-90.

12. Monnet X, Rienzo M, Osman D, et al. Passive leg raising predicts fluid responsiveness in the critically ill. Crit Care Med. 2006; 34:1402-1407.

13. Monnet X, Teboul JL. Passive leg raising. Intensive Care Med. 2008;34(4): 659-663.

14. Monnet X, Marik P, Teboul JL. Passive leg raising for predicting fluid responsiveness: a systematic review and meta-analysis. Intensive Care Med. 2016;42(12):1935-1947.

15. Thiel SW, Kollef MH, Isakow W. Non-invasive stroke volume measurement and passive leg raising predict volume responsiveness in medical ICU patients: an observational cohort study. Crit Care. 2009;13(4):R111.

16. Finfer S, Liu B, Taylor C, et al; SAFE TRIPS Investigators. Resuscitation fluid use in critically ill adults: an international cross sectional study in 391 intensive care units. Crit Care. 2010;14(5):R185.

17. Duus N, Shogilev DJ, Skibsted S, et al. The reliability and validity of passive leg raise and fluid bolus to assess fluid responsiveness in spontaneously breathing emergency department patients. J Crit Care. 2015;30(1):217.e1-e5.

18. Bentzer P, Griesdale DE, Boyd J, MacLean K, Sirounis D, Ayas NT. Will this hemodynamically unstable patient respond to a bolus of intravenous fluids? JAMA. 2016;316(12):1298-1309.

19. Cherpanath TG, Hirsch A, Geerts BF, et al. Predicting fluid responsiveness by passive leg raising: a systematic review and meta-analysis of 23 clinical trials. Crit Care Med. 2016;44(5):981-991. 
20. Lakhal K, Ehrmann S, Runge I, et al. Central venous pressure measurements improve the accuracy of leg raising-induced change in pulse pressure to predict fluid responsiveness. Intensive Care Med. 2010;36(6):940-948.

21. Kyriakides ZS, Koukoulas A, Paraskevaidis IA, et al. Does passive leg raising increase cardiac performance? A study using Doppler echocardiography. Int J Cardiol. 1994;44(3):288-293.

22. Zorko N, Kamenik M, Starc V. The effect of Trendelenburg position, lactated Ringer's solution and 6\% hydroxyethyl starch solution on cardiac output after spinal anesthesia. Anesth Analg. 2009;108(2):655-659.
23. Miyabe M, Sato S. The effect of head-down tilt position on arterial blood pressure after spinal anesthesia for cesarean delivery. Reg Anesth. 1997;22(3):239-242.

24. Frost H, Mortensen CR, Secher NH, Nielsen HB. Postoperative volume balance: does stroke volume increase in Trendelenburg's position? Clin Physiol Funct Imaging. 2015;37(3):314-316.

25. Reuter DA, Felbinger TW, Schmidt C, et al. Trendelenburg positioning after cardiac surgery: effects on intrathoracic blood volume index and cardiac performance. Eur J Anaesthesiol. 2003;20:17-20.
Integrated Blood Pressure Control

\section{Publish your work in this journal}

Integrated Blood Pressure Control is an international, peer-reviewed open-access journal focusing on the integrated approach to managing hypertension and risk reduction. Treating the patient and comorbidities together with diet and lifestyle modification and optimizing healthcare resources through a multidisciplinary team approach constitute key

\section{Dovepress}

features of the journal. This journal is indexed on American Chemical Society's Chemical Abstracts Service (CAS). The manuscript management system is completely online and includes a very quick and fair peerreview system, which is all easy to use. Visit http://www.dovepress.com/ testimonials.php to read real quotes from published authors.

Submit your manuscript here: https://www.dovepress.com/integrated-blood-pressure-control-journal 